\title{
A Quantum-chemical Study of the Relationships Between Electronic Structure and Anti-proliferative Activity of Quinoxaline Derivatives on the HeLa Cell Line
}

\author{
Gaston Assongba Kpotin ${ }^{1, ~ *, ~ J u a n ~ S e b a s t i a ́ n ~ G o ́ m e z-J e r i a ~}{ }^{2}$ \\ ${ }^{1}$ Department of Chemistry, Faculty of Sciences and Technologies, University of Abomey-Calavi, Abomey-Calavi, Republic of Benin \\ ${ }^{2}$ Quantum Pharmacology Unit, Department of Chemistry, Faculty of Sciences, University of Chile, Santiago, Chile
}

Email address:

gaston.kpotin@fast.uac.bj (G. A. Kpotin)

${ }^{*}$ Corresponding author

To cite this article:

Gaston Assongba Kpotin, Juan Sebastián Gómez-Jeria. A Quantum-chemical Study of the Relationships Between Electronic Structure and Anti-proliferative Activity of Quinoxaline Derivatives on the HeLa Cell Line. International Journal of Computational and Theoretical Chemistry. Vol. 5, No. 6, 2017, pp. 59-68. doi: 10.11648/j.ijctc.20170506.12

Received: November 28, 2017; Accepted: December 9, 2017; Published: January 11, 2018

\begin{abstract}
A study of the relationships between electronic structure and anti-proliferative activity of quinoxaline derivatives on the HeLa cell line was carried out. For this QSAR study the technique employed is the Klopman-Peradejordi-Gómez (KPG) method. We obtain a statistically significant equation $\left(R=0.97 \quad R^{2}=0.94\right.$ adj- $R^{2}=0.91 F(8,15)=29.50 p<0.000001$ and $\mathrm{SD}=0.06$ ). The results showed that the variation of the activity depends on the variation of the values of eight local atomic reactivity indices. The process seems to be charge and orbital-controlled. Based on the analysis of the result, a partial twodimensional pharmacophore was built. The results should be useful to propose new molecules which higher activity.
\end{abstract}

Keywords: Quinoxaline, HeLa Cell Line, KPG Method, QSAR, Pharmacophore, DFT

\section{Introduction}

Hela is the first immortalized cell line [1]. This cell line originates from a cervical cancer tumor of a patient named Henrietta Lacks, who later died of her cancer in 1951 [1]. One of the earliest uses of HeLa cells was to develop the vaccine against the polio virus [2]. The genomic and transcriptomic resource for a HeLa cell line based on deep DNA and RNA sequencing was created in 2013 [3]. Several studies are performed to found molecules that inhibit the proliferation of this cell line [4-28]. Theoretical studies were also done and are useful to explain the mechanism, the affinity and the activities of different compounds [26-29]. This work presents the results of the use of the KPG method [30] to obtain quantitative relationships between the electronic structure of quinoxaline derivatives and their antiproliferative activities on the HeLa cell line.

\section{Methods, Models and Calculations}

\subsection{Methods and Models}

For this study we use the Klopman-Peradejordi-Gomez (KPG) method. In 1967, Klopman and Hudson presented a general perturbation model for chemical reactivity including ionic interactions and not restricted only to $\pi$ electron [3133]. In their model, the electronic energy change, $\Delta \mathrm{E}$, associated with the interaction of atom $i$ of molecule A with atom $\mathrm{j}$ of molecule $\mathrm{B}$ is given by:

$$
\Delta E=\sum_{p}\left[Q_{i} Q_{j} / R_{i j}+(1 / 2)\left(\beta_{i j}^{2}\right) \sum_{m} \sum_{n} F_{m i} F_{n^{\prime} j} /\left(E_{m}-E_{n^{\prime}}\right)-(1 / 2)\left(\beta_{i j}^{2}\right) \sum_{m^{\prime}} \sum_{n} F_{m^{\prime} i} F_{n j} /\left(E_{m^{\prime}}-E_{n}\right)\right]
$$

where $Q_{i}$ is the net charge of atom $I, F_{m i}$ is the Fukui index of $\mathrm{OM} \mathrm{m}$ of atom $\mathrm{i}, \beta_{\mathrm{ij}}$ is the resonance integral (assumed to be independent of the kind of atomic orbitals (OA) because the A-B complex does not involve covalent bonds), $\mathrm{E}_{\mathrm{m}}\left(\mathrm{E}_{\mathrm{m}}{ }^{\prime}\right)$ 
is the energy of the m-th occupied MO ( $\mathrm{m}$ ' for the empty MOs) of molecule A. $\mathrm{n}$ and $\mathrm{n}$ ' refer to molecule B. The summation on $p$ is over all interacting atom pairs. The first term of the right side of Equation 1 represents the electrostatic interaction between atom with net charges $Q_{i}$ and $\mathrm{Q}_{\mathrm{j}}$. The next two terms introduce the interactions between occupied MOs of one molecule with the empty MOs of the other molecule and vice versa. As this model represents the interaction energy in terms of atom-atom interactions, it was only a matter of time that someone applied it for pharmacological/biological problems. Then, in 1971, Peradejordi et al published an article where they presented the results of a quantum-chemical study of the structure-activity relationships of tetracycline antibiotics [34]. The authors proposed that the inhibitory rate constants, $K_{i}^{I}$, can be expressed as:

$$
\log K_{i}^{I}=\text { cons } \tan t+\log K_{i}^{c}
$$

where $K_{i}^{c}$ is the ribosome-tetracycline equilibrium constant. Now, let us consider the state of thermodynamic equilibrium and a 1:1 stoichiometry in the formation of the drug-receptor complex:

$$
D_{i}+R \rightleftarrows D_{i} R
$$

where $D_{i}$ is the drug, $R$ the receptor and $D_{i} R$ the drugreceptor complex. According to statistical thermodynamics the equilibrium constant $K_{i}$ is written as:

$$
K_{i}=\frac{Q_{D_{i} R}}{Q_{D_{i}} Q_{R}} \exp \left(-\Delta \varepsilon_{0}^{i} / k T\right)
$$

where $Q_{D_{i} R}, Q_{D_{i}}$ and $Q_{R}$ are respectively the total partition functions of the drug-receptor complex, the drug and the receptor; $k$ is the Boltzmann's constant and $T$ is the absolute temperature. $\Delta \varepsilon_{0}^{i}$ is the difference between the ground-state energy of $D_{i} R$ and the energies of the groundstates of $D_{i}$ and $R$ :

$$
\Delta \varepsilon_{0}^{i}=\varepsilon_{D_{i} R}-\left(\varepsilon_{D_{i}}+\varepsilon_{R}\right)
$$

Peradejordi et al consider that the partition function terms and the solvation energy are constant. After overs considerations and approximations (for details see [34]), the linear equations is obtained:

$$
\log K_{i}^{I}=A+\sum_{p}\left\{a_{p} Q_{b, p}+b_{p} S_{b, p}^{E}+c_{p} S_{b, p}^{N}\right\} \mathrm{i}=1,2, \ldots, \mathrm{n}
$$

where $A, a_{p}, b_{p}, c_{p}$ are constant to be determined $Q_{b, p}$ is the net charge $S_{b, p}^{E}$ is the total atomic electrophilic superdelocalizability of atom $\mathrm{p}$ and $S_{b, p}^{N}$ is the total atomic nucleophilic superdelocalizability of atom p. Gómez-Jeria continued working the drug-site interaction energy and published the results [35-43]. In 2013, he derived the following equation [44]:

$$
\begin{aligned}
& \log \left(I C_{50}\right)=a+b M_{D_{i}}+c \log \left[\sigma_{D_{i}} /(A B C)^{1 / 2}\right]+\sum_{j}\left[e_{j} Q_{j}+f_{j} S_{j}^{E}+s_{j} S_{j}^{N}\right]+ \\
& \sum_{j} \sum_{m}\left[h_{j}(m) F_{j}(m)+x_{j}(m) S_{j}^{E}(m)\right]++\sum_{j} \sum_{m^{\prime}}\left[r_{j}\left(m^{\prime}\right) F_{j}\left(m^{\prime}\right)+t_{j}\left(m^{\prime}\right) S_{j}^{N}\left(m^{\prime}\right)\right] \\
& +\sum_{j}\left[g_{j} \mu_{j}+k_{j} \eta_{j}+o_{j} \omega_{j}+z_{j} \varsigma_{j}+w_{j} Q_{j}^{\max }\right]
\end{aligned}
$$

where a, b and c are constants, $M_{D_{i}}$ is the drug's mass, $\sigma_{D_{i}}$ its symmetry number and $A B C$ the product of the drug's moments of inertia about the three principal axes of rotation. $\mathrm{Q}_{\mathrm{j}}$ is the net charge of atom $\mathrm{j}, S_{j}^{E}$ and $S_{j}^{N}$ are, respectively, the total atomic electrophilic and nucleophilic superdelocalizabilities of Fukui et al., $\mathrm{F}_{\mathrm{j}, \mathrm{m}}\left(\mathrm{F}_{\mathrm{j}, \mathrm{m}}{ }^{\prime}\right)$ is Fukui index of the occupied (vacant) $\mathrm{MO} \mathrm{m}\left(\mathrm{m}^{\prime}\right)$ located on atom $\mathrm{j}$. $S_{j}^{E}(m)$ is the atomic electrophilic superdelocalizability of MO $\mathrm{m}$ on atom $\mathrm{j}$, etc. The total atomic electrophilic superdelocalizability of atom $\mathrm{j}$ corresponds to the sum over occupied MOs of the $S_{j}^{E}(m)$ 's and the total atomic nucleophilic superdelocalizability of atom $\mathrm{j}$ is the sum over vacant MOs of $S_{j}^{N}\left(m^{\prime}\right)$ 's. $\mu_{j}$ is the local atomic electronic chemical potential of atom $\mathrm{j}, \eta_{j}$ is the local atomic hardness of atom $\mathrm{j}, \omega_{j}$ is the local atomic electrophilicity of atom $\mathrm{j}$, $\varsigma_{j}$ is the local atomic softness of atom $\mathrm{j}$, and $Q_{j}^{\max }$ is the maximum amount of electronic charge that atom $\mathrm{j}$ may accept from another site. $\mathrm{O}_{k}$ 's are the orientational parameters of the substituents. Throughout this paper $\mathrm{HOMO}_{\mathrm{j}}{ }^{*}$ refers to the highest occupied molecular orbital localized on atom $\mathrm{j}$ and $\mathrm{LUMO}_{\mathrm{j}}{ }^{*}$ to the lowest empty MO localized on atom $\mathrm{j}$.

$$
\text { with } \log \left[(A B C)^{-1 / 2}\right] \simeq \sum_{t} \sum_{t} m_{i, t} R_{i, t}^{2}=\sum_{t} O_{t}
$$

where the summation over $\mathrm{t}$ is over the different substituents of the molecule, $m_{i, t}$ is the mass of the $\mathrm{i}$-th atom belonging to the t-th substituent, $R_{i, t}$ being its distance to the atom to which the substituent is attached. This approximation allows him to transform a molecular property into a sum of substituent properties. He proposed that these terms represent 
the fraction of molecules attaining the proper orientation to interact with a given site. He called them Orientational Parameters (OP). The new local atomic reactivity indices (LARIs) of Eq. 7 are defined as follows:

Local atomic electronic chemical potential:

$$
\mu_{i}=\left(\varepsilon_{H_{O M O}^{*}, i}+\varepsilon_{L U M O^{*}, i}\right) / 2
$$

Local atomic hardness:

$$
\eta_{i}=\left(\varepsilon_{H O M O *, i}-\varepsilon_{L U M O^{*}, i}\right)
$$

Local electrophilic superdelocalizability of the HOMO* of atom $\mathrm{i}$ and local nucleophilic superdelocalizability of the LUMO* of atom i:

$$
\begin{gathered}
S_{i}^{E^{*}}=\frac{F_{i, H O M O^{*}}}{\varepsilon_{\text {HOMO* }}} \\
S_{i}^{N^{*}}=\frac{F_{i, L U M O^{*}}}{\varepsilon_{\text {LUMO* }}}
\end{gathered}
$$

Local atomic softness of atom i:

$$
S_{i}=\frac{1}{\eta_{i}}
$$

Local atomic electrophilicity of atom i:

$$
\omega_{i}=\frac{\mu_{i}^{2}}{2 \eta_{i}}
$$

The maximal amount of charge atom i may receive:

\begin{tabular}{|c|c|c|}
\hline Index & Name & Physical meaning \\
\hline$Q_{i}$ & Net atomic charge of atom $i$ & Electrostatic interaction \\
\hline$S_{i}^{E}$ & $\begin{array}{l}\text { Total atomic electrophilic } \\
\text { superdelocalizability of atom i }\end{array}$ & $\begin{array}{l}\text { Total atomic electron-donating } \\
\text { capacity of atom i } \\
\text { (MO-MO interaction) }\end{array}$ \\
\hline$S_{i}^{N}$ & $\begin{array}{l}\text { Total atomic nucleophilic } \\
\text { superdelocalizability of atom i }\end{array}$ & $\begin{array}{l}\text { Total atomic electron-accepting } \\
\text { capacity of atom i } \\
\text { (MO-MO interaction) }\end{array}$ \\
\hline$S_{i}^{E}(m)$ & $\begin{array}{l}\text { Orbital atomic electrophilic } \\
\text { superdelocalizability of atom } \\
\text { i and occupied MO m }\end{array}$ & $\begin{array}{l}\text { Electron-donating capacity } \\
\text { of atom i at occupied MO m } \\
\text { (MO-MO interaction) }\end{array}$ \\
\hline$S_{i}^{N}\left(m^{\prime}\right)$ & $\begin{array}{l}\text { Orbital atomic nucleophilic } \\
\text { superdelocalizability of atom } \\
\text { i and empty MO m' }\end{array}$ & $\begin{array}{l}\text { Electron-accepting capacity } \\
\text { of atom i at empty MO m' } \\
\text { (MO-MO interaction) }\end{array}$ \\
\hline$F_{i}$ & Fukui index of atom i & $\begin{array}{l}\text { Total electron population of atom i } \\
\text { (MO-MO interaction) }\end{array}$ \\
\hline$F_{m i}$ & $\begin{array}{l}\text { Fukui index of atom } \mathrm{i} \text { and } \\
\text { occupied MO m. }\end{array}$ & $\begin{array}{l}\text { Electron population of occupied MO } \\
\mathrm{m} \text { at atom i } \\
\text { (MO-MO interaction) }\end{array}$ \\
\hline$F_{m^{\prime} i}$ & $\begin{array}{l}\text { Fukui index of atom i and } \\
\text { empty MO m' }\end{array}$ & $\begin{array}{l}\text { Electron population of empty MO } \\
\text { m' at atom i } \\
\text { (MO-MO interaction) }\end{array}$ \\
\hline$\mu_{i}$ & $\begin{array}{l}\text { Local atomic electronic } \\
\text { chemical potential of atom i }\end{array}$ & $\begin{array}{l}\text { Propensity of atom i to gain or } \\
\text { lose electrons }\end{array}$ \\
\hline$\eta_{i}$ & Local atomic hardness of atom i & $\begin{array}{l}\text { Resistance of atom i to exchange } \\
\text { electrons with a site }\end{array}$ \\
\hline$\varsigma_{i}$ & Local atomic softness of atom i & The inverse of $\mu_{i}$ \\
\hline$\omega_{i}$ & $\begin{array}{l}\text { Local atomic electrophilicity } \\
\text { of atom i }\end{array}$ & $\begin{array}{l}\text { Propensity of atom i to receive } \\
\text { extra electronic charge together with } \\
\text { its resistance to exchange charge } \\
\text { with a site }\end{array}$ \\
\hline$Q_{i}^{\max }$ & $\begin{array}{l}\text { Maximal amount of electronic } \\
\text { charge atom i may receive }\end{array}$ & $\begin{array}{l}\text { Maximal amount of electronic charge } \\
\text { that atom i may receive from a donor site }\end{array}$ \\
\hline
\end{tabular}

$$
Q_{i}^{\max }=-\frac{\mu_{i}}{\eta_{i}}
$$

The physical meaning of these indices is summarized in Table 1 .

Table 1. Local Atomic Reactivity Indices and their physical meaning [44].

The Klopman-Peradejordi-Gómez (KPG) method is also discussed in many previous papers $[30,35,36,38,39,42$, 44-46]. From a conceptual perspective, the work presented here is a test of the hypothesis stating that the KPG model can provide a quantitative and formal relationship between the molecular structure and any biological activity. Nowadays, the KPG model produced excellent results in all its applications[35, 44, 46-53].

\subsection{Selection of Molecules}

For this study, a series of quinoxline derivatives were selected [23]. These molecules have an anti-proliferative activity on the HeLa cell line. The experimental data was taken from a recent study [23]. The structures of the 
compounds are shown in Figure 1 and Table 2 which also summarizes the values of their median inhibitory

concentrations expressed as $\log \left(\mathrm{IC}_{50}\right)$.

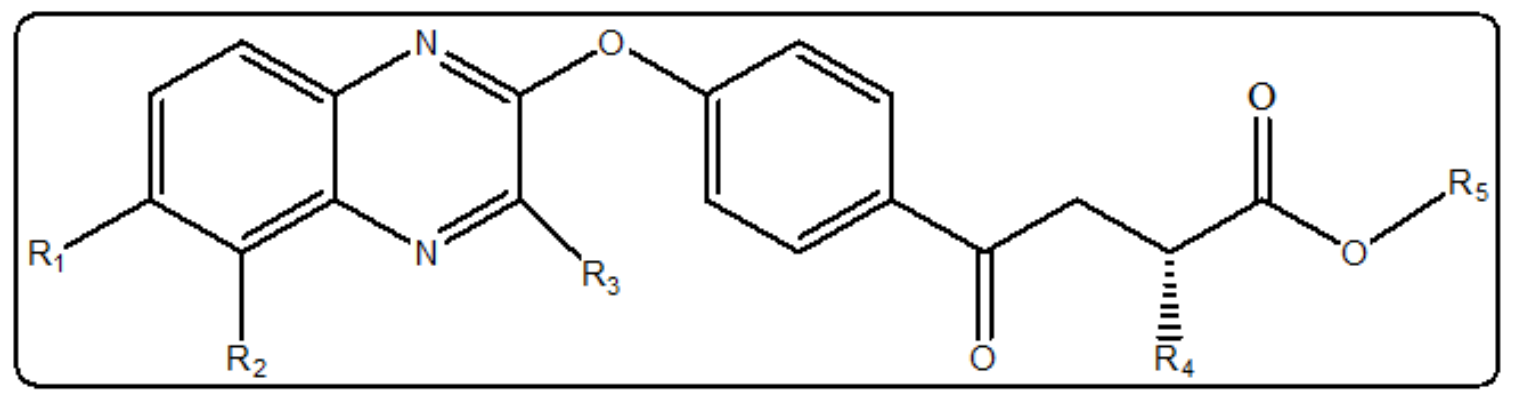

Figure 1. Structure of quinoxaline derivatives.

Table 2. Quinoxalines and their experimental anti-proliferative activity.

\begin{tabular}{|c|c|c|c|c|c|c|}
\hline Mol. & $\mathbf{R}_{1}$ & $\mathbf{R}_{2}$ & $\mathbf{R}_{3}$ & $\mathbf{R}_{4}$ & $\mathbf{R}_{5}$ & $\log \left(\mathrm{IC}_{50}\right)$ \\
\hline 1 & $\mathrm{H}$ & $\mathrm{H}$ & $\mathrm{CH}_{3}$ & $\mathrm{CH}_{3}$ & $\mathrm{CH}_{3}$ & 1.66 \\
\hline 2 & $\mathrm{H}$ & $\mathrm{H}$ & $\mathrm{CH}_{3}$ & $\left(\mathrm{CH}_{3}\right)_{2} \mathrm{CHCH}_{2-}$ & $\mathrm{CH}_{3}$ & 1.35 \\
\hline 3 & $\mathrm{H}$ & $\mathrm{H}$ & $\mathrm{CH}_{3}$ & $\mathrm{CH}_{3} \mathrm{CH}_{2} \mathrm{CH}\left(\mathrm{CH}_{3}\right)-$ & $\mathrm{CH}_{3}$ & 1.58 \\
\hline 4 & $\mathrm{H}$ & $\mathrm{Cl}$ & $\mathrm{CH}_{3}$ & $\mathrm{CH}_{3}$ & $\mathrm{CH}_{3}$ & 1.42 \\
\hline 5 & $\mathrm{H}$ & $\mathrm{Cl}$ & $\mathrm{CH}_{3}$ & $\left(\mathrm{CH}_{3}\right)_{2} \mathrm{CHCH}_{2-}$ & $\mathrm{CH}_{3}$ & 1.51 \\
\hline 6 & $\mathrm{Cl}$ & $\mathrm{H}$ & $\mathrm{CH}_{3}$ & $\mathrm{C}_{6} \mathrm{H}_{5} \mathrm{CH}_{2^{-}}$ & $\mathrm{CH}_{3}$ & 1.44 \\
\hline 7 & $\mathrm{Cl}$ & $\mathrm{H}$ & $\mathrm{CH}_{3}$ & $\left(\mathrm{CH}_{3}\right)_{2} \mathrm{CHCH}_{2-}$ & $\mathrm{CH}_{3}$ & 1.34 \\
\hline 8 & $\mathrm{H}$ & $\mathrm{H}$ & $\mathrm{CH}_{3}$ & $\mathrm{CH}_{3}$ & $\mathrm{H}$ & 1.45 \\
\hline 9 & $\mathrm{H}$ & $\mathrm{H}$ & $\mathrm{CH}_{3}$ & $\left(\mathrm{CH}_{3}\right)_{2} \mathrm{CHCH}_{2-}$ & $\mathrm{H}$ & 1.29 \\
\hline 10 & $\mathrm{H}$ & $\mathrm{H}$ & $\mathrm{CH}_{3}$ & $\mathrm{CH}_{3} \mathrm{CH}_{2} \mathrm{CH}\left(\mathrm{CH}_{3}\right)-$ & $\mathrm{H}$ & 1.48 \\
\hline 11 & $\mathrm{H}$ & $\mathrm{Cl}$ & $\mathrm{CH}_{3}$ & $\mathrm{CH}_{3}$ & $\mathrm{H}$ & 1.32 \\
\hline 12 & $\mathrm{H}$ & $\mathrm{Cl}$ & $\mathrm{CH}_{3}$ & $\left(\mathrm{CH}_{3}\right)_{2} \mathrm{CHCH}_{2^{-}}$ & $\mathrm{H}$ & 1.33 \\
\hline 13 & $\mathrm{Cl}$ & $\mathrm{H}$ & $\mathrm{CH}_{3}$ & $\mathrm{C}_{6} \mathrm{H}_{5} \mathrm{CH}_{2^{-}}$ & $\mathrm{H}$ & 1.33 \\
\hline 14 & $\mathrm{Cl}$ & $\mathrm{H}$ & $\mathrm{CH}_{3}$ & $\left(\mathrm{CH}_{3}\right)_{2} \mathrm{CHCH}_{2-}$ & $\mathrm{H}$ & 1.16 \\
\hline 15 & $\mathrm{H}$ & $\mathrm{H}$ & $\mathrm{NH}\left(\mathrm{CH}_{2}\right)_{11} \mathrm{CH}_{3}$ & $\mathrm{C}_{6} \mathrm{H}_{5} \mathrm{CH}_{2-}$ & $\mathrm{CH}_{3}$ & 1.48 \\
\hline 16 & $\mathrm{H}$ & $\mathrm{H}$ & $\mathrm{NH}\left(\mathrm{CH}_{2}\right)_{11} \mathrm{CH}_{3}$ & $\mathrm{CH}_{3}$ & $\mathrm{CH}_{3}$ & 1.43 \\
\hline 17 & $\mathrm{H}$ & $\mathrm{H}$ & $\mathrm{NH}\left(\mathrm{CH}_{2}\right)_{11} \mathrm{CH}_{3}$ & $\left(\mathrm{CH}_{3}\right)_{2} \mathrm{CHCH}_{2^{-}}$ & $\mathrm{CH}_{3}$ & 1.49 \\
\hline 18 & $\mathrm{H}$ & $\mathrm{H}$ & $\mathrm{NH}\left(\mathrm{CH}_{2}\right)_{11} \mathrm{CH}_{3}$ & $\mathrm{H}$ & $\mathrm{CH}_{3}$ & 1.63 \\
\hline 19 & $\mathrm{H}$ & $\mathrm{H}$ & $\mathrm{NHC}\left(\mathrm{CH}_{2}\right)_{3}$ & $\mathrm{CH}_{3}$ & $\mathrm{CH}_{3}$ & 1.59 \\
\hline 20 & $\mathrm{H}$ & $\mathrm{H}$ & $\mathrm{NHC}\left(\mathrm{CH}_{2}\right)_{3}$ & $\mathrm{C}_{6} \mathrm{H}_{5} \mathrm{CH}_{2^{-}}$ & $\mathrm{CH}_{3}$ & 1.01 \\
\hline 21 & $\mathrm{H}$ & $\mathrm{H}$ & $\mathrm{NH}\left(\mathrm{CH}_{2}\right)_{11} \mathrm{CH}_{3}$ & $\mathrm{C}_{6} \mathrm{H}_{5} \mathrm{CH}_{2^{-}}$ & $\mathrm{H}$ & 0.87 \\
\hline 22 & $\mathrm{H}$ & $\mathrm{H}$ & $\mathrm{NH}\left(\mathrm{CH}_{2}\right)_{11} \mathrm{CH}_{3}$ & $\mathrm{CH}_{3}$ & $\mathrm{H}$ & 0.52 \\
\hline 23 & $\mathrm{H}$ & $\mathrm{H}$ & $\mathrm{NH}\left(\mathrm{CH}_{2}\right)_{11} \mathrm{CH}_{3}$ & $\left(\mathrm{CH}_{3}\right)_{2} \mathrm{CHCH}_{2-}$ & $\mathrm{H}$ & 1.33 \\
\hline 24 & $\mathrm{H}$ & $\mathrm{H}$ & $\mathrm{NH}\left(\mathrm{CH}_{2}\right)_{11} \mathrm{CH}_{3}$ & $\mathrm{H}$ & $\mathrm{H}$ & 1.42 \\
\hline 25 & $\mathrm{H}$ & $\mathrm{H}$ & $\mathrm{NHC}\left(\mathrm{CH}_{2}\right)_{3}$ & $\mathrm{CH}_{3}$ & $\mathrm{H}$ & 1.26 \\
\hline 26 & $\mathrm{H}$ & $\mathrm{H}$ & $\mathrm{NHC}\left(\mathrm{CH}_{2}\right)_{3}$ & $\mathrm{CH}_{3} \mathrm{CH}_{2} \mathrm{CH}\left(\mathrm{CH}_{3}\right)-$ & $\mathrm{H}$ & 1.63 \\
\hline 27 & $\mathrm{H}$ & $\mathrm{H}$ & $\mathrm{NHC}\left(\mathrm{CH}_{2}\right)_{3}$ & $\mathrm{C}_{6} \mathrm{H}_{5} \mathrm{CH}_{2-}$ & $\mathrm{H}$ & 1.18 \\
\hline
\end{tabular}

\subsection{Calculations}

The electronic structure of each fully optimized molecule was obtained using the Density Functional Theory (DFT) at the B3LYP/6-31G (d, p) level with the Gaussian software [54]. The local atomic reactivity indices were calculated from the single point results of Gaussian03 using the D-CentQSAR software [55] with a correction for Mulliken populations [56]. All populations of electrons less than or equal to $0.01 \mathrm{e}$ are considered null [56]. The orientational parameters of the substituents are calculated in the usual manner [57, 58]. We have used the concept of common skeleton defined as a set of atoms common to all the molecules analyzed. We hypothesize that the variation of the numerical values of the local atomic reactivity indices (LARIs) of the atoms of this common skeleton accounts for almost all the variation of the biological activity. As the number of LARIs involved is greater that the number of molecules, the solving of the linear systems of equations is not possible. For this reason we employed the technique of multiple linear regression analysis (LMRA) to determine the 
atoms that are directly involved in the variation of the biological activity. The data matrix contains $\log \left(\mathrm{IC}_{50}\right)$ as a dependent variable, and the local indices of atomic reactivity of all the atoms of the common skeleton as independent variables. The Statistica 10 software was used to perform LMRA studies [59]. The numbering of the common skeleton atoms is shown in Figure. 2.

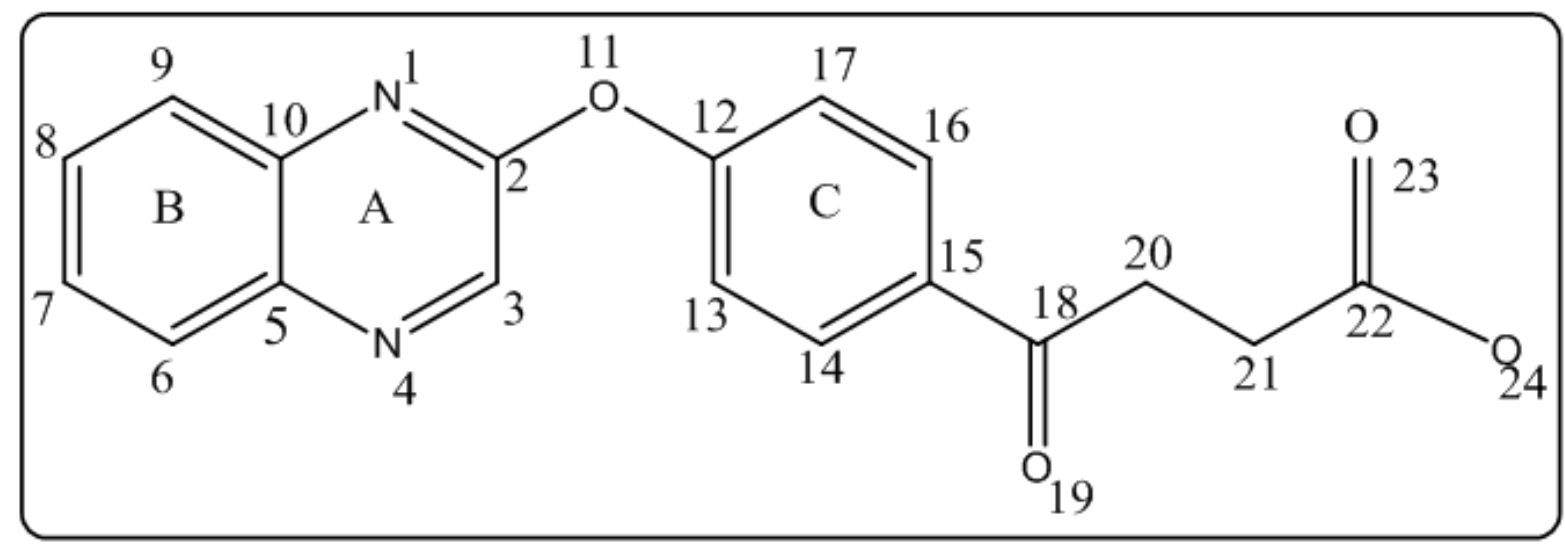

Figure 2. Common skeleton numbering.

\section{Results}

The best statistically significant equation obtained is the following:

$$
\begin{aligned}
\log \left(I_{50}\right)= & -33.32+1.69 F_{21}\left(H_{O M O}\right)^{*}-2.61 S_{21}^{E}+0.04 S_{16}^{N}-78.05 Q_{16}-0.12 F_{23}\left(\text { HOMO }^{*}\right. \\
& -1.28 F_{15}(\text { LUMO })^{*}+0.69 F_{20}\left(\text { HOMO }^{*}+0.001 S_{22}^{N}\left(\text { LUMO }^{*}\right.\right.
\end{aligned}
$$

with $\mathrm{n}=24, \mathrm{R}=0.97, \mathrm{R}^{2}=0.94, \operatorname{adj}-\mathrm{R}^{2}=0.91, \mathrm{~F}(8.15)=29.50$, $(p<0.000001)$ and a standard error of estimate of 0.06 . No outliers were detected and no residuals fall outside the $\pm 2 \sigma$ limits. Here $F_{21}(H O M O) *$ is the electron population (Fukui index) of the highest occupied MO localized on atom $21, S_{21}^{E}$ is the total atomic electrophilic superdelocalizability of atom $21, S_{16}^{N}$ is the total atomic nucleophilic superdelocalizability of atom $16, Q_{16}$ is the net charge of atom $16, F_{23}(H O M O) *$ is the Fukui index of the highest occupied MO localized on atom $23, F_{15}(L U M O) *$ is the Fukui index of the first lowest vacant $\mathrm{MO}$ localized on atom $15, F_{20}(H O M O)^{*}$ is the electron population of the highest occupied MO localized on atom 20 and $S_{22}^{N}(L U M O) *$ is the atomic nucleophilic superdelocalizability of the first lowest vacant MO localized on atom 22. Table 3 shows the beta coefficients and the t-test results for the significance of coefficients of equation 1 . Concerning independent variables, Table 4 shows that the highest internal correlation is $\mathrm{r}^{2}\left[\mathrm{~F}_{20}(\mathrm{HOMO}) *\right.$, $\left.\mathrm{F}_{21}(\mathrm{HOMO})^{*}\right]=0.43$. Figure 3 shows the plot of observed values vs. calculated values of $\log \left(\mathrm{IC}_{50}\right)$. The associated statistical parameters of Eq.16 show that this equation is statistically significant and that the variation of the numerical values of eight LARIs explains about $91 \%$ of the variation of the variation of the biological activity.

Table 3. Beta coefficients and t-test for significance of coefficients in equation 1.

\begin{tabular}{llll}
\hline & Beta & $\mathbf{t}(\mathbf{1 0})$ & p-level \\
\hline$F_{21}(H O M O)^{*}$ & 0.70 & 7.44 & $<0.000002$ \\
$S_{21}^{E}$ & -1.11 & -10.08 & $<0.000000$ \\
$S_{16}^{N}$ & 0.97 & 7.67 & $<0.000001$ \\
$Q_{16}$ & -0.43 & -5.06 & $<0.0001$ \\
$F_{23}(H O M O)^{*}$ & -0.26 & -3.51 & $<0.003$ \\
$F_{15}(L U M O)^{*}$ & -0.31 & -3.26 & $<0.005$ \\
$F_{20}(H O M O)^{*}$ & 0.34 & 3.23 & $<0.006$ \\
$S_{22}^{N}(L U M O)^{*}$ & 0.28 & 2.61 & $<0.02$ \\
\hline
\end{tabular}




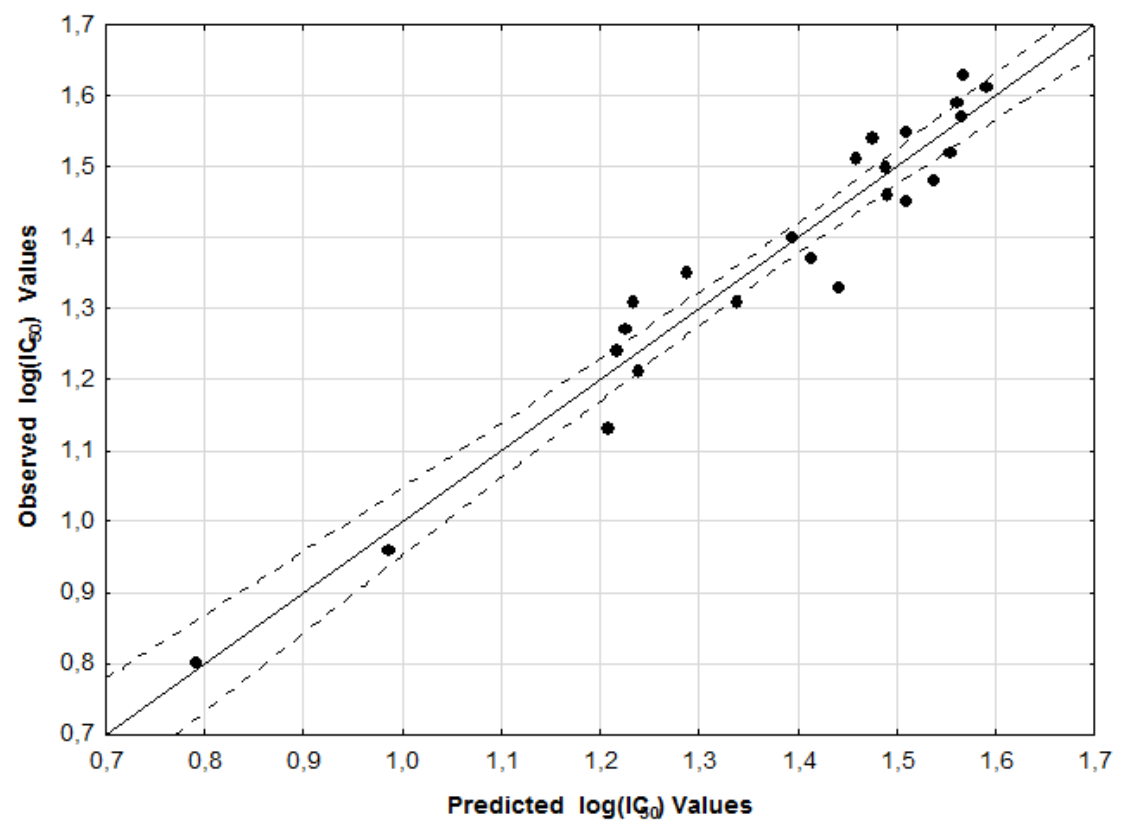

Figure 3. Plot of predicted vs. observed $\log (I C 50)$ values. Dashed lines denote the 95\% confidence interval.

Table 4. Squared correlation coefficients for the variables appearing in equation 16.

\begin{tabular}{llllllll}
\hline & $\boldsymbol{F}_{21}(\boldsymbol{H O M O})^{*}$ & $\boldsymbol{S}_{21}^{E}$ & $\boldsymbol{S}_{16}^{N}$ & $\boldsymbol{Q}_{16}$ & $\boldsymbol{F}_{23}(\boldsymbol{H O M O})^{*}$ & $\boldsymbol{F}_{15}(\boldsymbol{L U M O})^{*}$ & $\boldsymbol{F}_{20}(\boldsymbol{H O M O})^{*}$ \\
\hline$S_{21}^{E}$ & 0.004 & 1.00 & & & & \\
$S_{16}^{N}$ & 0.02 & 0.3 & 1.00 & & & \\
$Q_{16}$ & 0.002 & 0.034 & 0.2 & 1.00 & & \\
$F_{23}(H O M O)^{*}$ & 0.08 & 0.05 & 0.1 & 0.0001 & 1.00 & & \\
$F_{15}(L U M O) *$ & 0.001 & 0.03 & 0.4 & 0.07 & 0.03 & 0.002 & 1.00 \\
$F_{20}(H O M O)^{*}$ & 0.4 & 0.06 & 0.00004 & 0.03 & 0.01 & 0.19 & 0.02 \\
$S_{22}^{N}(L U M O)^{*}$ & 0.01 & 0.3 & 0.3 & 0.2 & 0.04 & \\
\hline
\end{tabular}

Local Molecular Orbitals

Tables 5 and 6 show the Local Molecular Orbitals of atom 5, 10, 20, 21, 22 and 23 (see Figure 3). Nomenclature: Molecule $(\mathrm{HOMO}) /(\mathrm{HOMO}-2) *(\mathrm{HOMO}-1) *(\mathrm{HOMO}) *-(\mathrm{LUMO}) *(\mathrm{LUMO}+1) *(\mathrm{LUMO}+2) *$.

Table 5. Local Molecular Orbitals of atoms 10, 15 and 20.

\begin{tabular}{|c|c|c|c|}
\hline Mol & Atom $10(C)$ & Atom $15(\mathrm{C})$ & Atom $20(C)$ \\
\hline $1(96)$ & $92 \pi 93 \pi 96 \pi-97 \pi 98 \pi 99 \pi$ & $94 \pi 95 \pi 96 \pi-97 \pi 98 \pi 100 \pi$ & $86 \sigma 90 \sigma 94 \sigma-103 \sigma 107109$ \\
\hline $2(108)$ & $104 \pi 105 \sigma 108 \pi-109 \pi 110 \pi 111 \pi$ & $106 \sigma 107 \pi 108 \pi-109 \pi 110 \pi 112 \pi$ & $99 \sigma 100 \sigma 106 \sigma-114 \sigma 115 \sigma 118 \sigma$ \\
\hline $3(116)$ & $113 \sigma 115 \pi 116 \pi-117 \pi 118 \pi 119 \pi$ & $114 \sigma 115 \pi 116 \pi-117 \pi 118 \pi 120$ & $107112 \sigma 114 \sigma-122 \sigma 124 \sigma 127$ \\
\hline $4(104)$ & $101 \sigma 103 \pi 104 \pi-105 \pi 106 \pi 107 \pi$ & $102 \sigma 103 \pi 104 \pi-105 \pi 106 \pi 108 \pi$ & $93 \sigma 98 \sigma 102 \sigma-112 \sigma 115 \sigma 118 \sigma$ \\
\hline $5(116)$ & $113 \sigma 115 \pi 116 \pi-117 \pi 118 \pi 119 \pi$ & $114 \sigma 115 \pi 116 \pi-117 \pi 118 \pi 120 \pi$ & $107 \sigma 111 \sigma 114 \sigma-122 \sigma 124 \sigma 127 \sigma$ \\
\hline $6(124)$ & $118 \sigma 119 \sigma 122 \pi-125 \pi 126 \pi 127 \pi$ & $120 \sigma 121 \pi 122 \pi-125 \pi 126 \pi 128 \pi$ & $120 \sigma 121 \sigma 124 \sigma-130 \sigma 132 \sigma 134 \sigma$ \\
\hline $7(116)$ & $112 \sigma 113 \pi 116 \pi-117 \pi 118 \pi 119 \pi$ & $114 \pi 115 \sigma 116 \pi-117 \pi 118 \pi 120 \pi$ & $108 \sigma 111 \sigma 115 \sigma-122 \sigma 124 \sigma 127 \sigma$ \\
\hline $8(92)$ & $88 \pi 89 \sigma 92 \pi-93 \pi 94 \pi 95 \pi$ & $90 \sigma 91 \pi 92 \pi-93 \pi 94 \pi 96 \pi$ & $81 \sigma 86 \sigma 90 \sigma-99 \sigma 100 \sigma 106 \sigma$ \\
\hline $9(104)$ & $100 \pi 101 \sigma 104 \pi-105 \pi 106 \pi 107 \pi$ & $102 \sigma 103 \pi 104 \pi-105 \pi 106 \pi 108 \pi$ & $98 \sigma 101 \sigma 102 \sigma-109 \sigma 110 \sigma 111 \sigma$ \\
\hline $10(104)$ & $100 \pi 101 \sigma 104 \pi-105 \pi 106 \pi 107 \pi$ & $102 \sigma 103 \pi 104 \pi-105 \pi 106 \pi 108 \pi$ & $97 \sigma 98 \sigma 102 \sigma-111 \sigma 112 \sigma 116 \sigma$ \\
\hline $11(100)$ & $97 \sigma 99 \pi 100 \pi-101 \pi 102 \pi 103 \pi$ & $98 \sigma 99 \pi 100 \pi-101 \pi 102 \pi 104 \pi$ & $89 \sigma 94 \sigma 98 \sigma-108 \sigma 110 \sigma 115 \sigma$ \\
\hline $12(112)$ & $109 \sigma 111 \pi 112 \pi-113 \pi 114 \pi 115 \pi$ & $110 \sigma 111 \pi 112 \pi-113 \pi 114 \pi 116 \pi$ & $104 \sigma 106 \sigma 110 \sigma-118 \sigma 120 \sigma 121 \sigma$ \\
\hline $13(120)$ & $114 \sigma 115 \sigma 118 \pi-121 \pi 122 \pi 123 \pi$ & $116 \sigma 117 \pi 118 \pi-121 \pi 122 \pi 124 \pi$ & $110 \sigma 112 \sigma 116 \sigma-126 \sigma 128 \sigma 130$ \\
\hline $14(112)$ & $109 \pi 110 \pi 112 \pi-113 \pi 114 \pi 115 \pi$ & $110 \pi 111 \sigma 112 \pi-113 \pi 114 \pi 116 \pi$ & $106110 \sigma 111 \sigma-118 \sigma 120 \sigma 122 \sigma$ \\
\hline $15(164)$ & $160 \sigma 162 \pi 164 \pi-168 \pi 17 \pi 77 \pi$ & $160 \pi 162 \pi 164 \pi-165 \pi 166 \pi 167 \pi$ & $158 \sigma 159 \sigma 163 \sigma-169 \sigma 171 \sigma 173 \sigma$ \\
\hline $16(144)$ & $142 \sigma 143 \pi 144 \pi-148 \pi 150 \pi 159 \sigma$ & $142 \pi 143 \pi 144 \pi-145 \pi 146 \pi 147 \pi$ & $125 \sigma 138 \sigma 141 \sigma-151 \sigma 157 \sigma 163 \sigma$ \\
\hline $17(156)$ & $154 \sigma 155 \pi 156 \pi-160 \pi 162 \pi 171 \pi$ & $154 \sigma 155 \pi 156 \pi-157 \pi 158 \pi 159 \pi$ & $145 \sigma 153 \sigma 154 \sigma-161 \sigma 163 \sigma 166 \sigma$ \\
\hline $18(140)$ & $138 \sigma 139 \pi 140 \pi-144 \pi 146 \pi 167$ & $138 \pi 139 \pi 140 \pi-141 \pi 142 \pi 143 \pi$ & $119 \sigma 134 \sigma 137 \sigma-147 \sigma 154 \sigma 161 \sigma$ \\
\hline $19(112)$ & $109 \sigma 111 \pi 112 \pi-116 \pi 118 \pi 122 \sigma$ & $110 \pi 111 \pi 112 \pi-113 \pi 114 \pi 115 \pi$ & $106 \sigma 108 \sigma 110 \sigma-119 \sigma 127 \sigma 128 \sigma$ \\
\hline
\end{tabular}




\begin{tabular}{llll}
\hline Mol & Atom 10 (C) & Atom 15 (C) & Atom 20 (C) \\
\hline $20(136)$ & $132 \sigma 134 \pi 136 \pi-140 \pi 143 \pi 144 \pi$ & $132 \pi 134 \pi 136 \pi-137 \pi 138 \pi 139 \pi$ & $131 \sigma 132 \sigma 135 \sigma-137 \sigma 141 \sigma 143 \sigma$ \\
$21(160)$ & $158 \pi 159 \pi 160 \pi-164 \pi 168 \pi 174 \sigma$ & $156 \pi 158 \pi 160 \pi-161 \pi 162 \pi 163 \pi$ & $152154 \sigma 155 \sigma-165 \sigma 167 \sigma 169 \sigma$ \\
$22(140)$ & $138 \sigma 139 \pi 140 \pi-144 \pi 146 \pi 155 \sigma$ & $138 \pi 139 \pi 140 \pi-141 \pi 142 \pi 143 \pi$ & $134 \sigma 136 \sigma 137 \sigma-147 \sigma 148 \sigma 162$ \\
$23(152)$ & $150 \sigma 151 \pi 152 \pi-156 \pi 158 \pi 168 \sigma$ & $150 \pi 151 \pi 152 \pi-153 \pi 154 \pi 155 \pi$ & $146 \sigma 148 \sigma 149 \sigma-157 \sigma 159 \sigma 160 \sigma$ \\
$24(136)$ & $134 \sigma 135 \pi 136 \pi-140 \pi 142 \pi 148 \sigma$ & $134 \pi 135 \pi 136 \pi-137 \pi 138 \pi 139 \pi$ & $132 \sigma 133 \sigma 134 \sigma-143 \sigma 144 \sigma 157 \sigma$ \\
$25(108)$ & $106 \sigma 107 \pi 108 \pi-112 \pi 114 \pi 119 \sigma$ & $106 \pi 107 \pi 108 \pi-109 \pi 110 \pi 111 \pi$ & $102 \sigma 104 \sigma 105 \sigma-115 \sigma 117 \sigma 123 \sigma$ \\
$26(120)$ & $118 \sigma 119 \pi 120 \pi-124 \pi 126 \pi 133 \sigma$ & $118 \pi 119 \pi 120 \pi-121 \pi 122 \pi 123 \pi$ & $114 \sigma 116 \sigma 117 \sigma-125 \sigma 127 \sigma 129 \sigma$ \\
$27(128)$ & $124 \sigma 126 \pi 128 \pi-132 \pi 136 \pi 141 \sigma$ & $124 \pi 126 \pi 128 \pi-129 \pi 130 \pi 131 \pi$ & $120 \sigma 122 \sigma 123 \sigma-133 \sigma 135 \sigma 137 \sigma$ \\
\hline
\end{tabular}

Table 6. Local Molecular Orbitals of atoms 21, 22 and 23.

\begin{tabular}{|c|c|c|c|}
\hline Mol. & Atom 21 (C) & Atom $22(C)$ & Atom $23(0)$ \\
\hline $1(96)$ & $86 \sigma 90 \sigma 94 \sigma-102 \sigma 105 \sigma 110 \sigma$ & $86 \pi 89 \pi 90 \pi-102 \pi 103 \pi 105 \pi$ & $89 \pi 90 \pi 94 \pi-102 \pi 111 \pi 138 \pi$ \\
\hline $2(108)$ & $102 \sigma 103 \sigma 106 \sigma-114 \sigma 120 \sigma 124 \sigma$ & $94 \sigma 101 \pi 102 \pi-113 \pi 114 \pi 115 \pi$ & $102 \pi 103 \pi 106 \pi-114 \pi 145 \pi 162 \pi$ \\
\hline $3(116)$ & $111 \sigma 112 \sigma 114 \sigma-122 \sigma 129 \sigma 130 \sigma$ & $109 \pi 111 \pi 112 \pi-122 \pi 124 \sigma 126 \sigma$ & $111 \pi 112 \pi 114 \pi-122 \pi 171 \pi 173 \pi$ \\
\hline $4(104)$ & $93 \sigma 98 \sigma 102 \sigma-110 \sigma 114 \sigma 119 \sigma$ & $93 \sigma 97 \pi 98 \pi-110 \pi 112 \pi 114 \sigma$ & $98 \pi 100 \pi 102 \pi-110 \pi 120 \sigma 147 \sigma$ \\
\hline $5(116)$ & $107 \sigma 111 \sigma 114 \sigma-122 \sigma 128 \sigma 133 \sigma$ & $102 \sigma 109 \pi 111 \pi-122 \pi 124 \pi 126 \sigma$ & $111 \pi 112 \pi 114 \pi-122 \pi 171 \pi 173 \pi$ \\
\hline $6(124)$ & $117 \sigma 120 \sigma 124 \sigma-130 \sigma 131 \sigma 132 \sigma$ & $115 \pi 117 \pi 120 \sigma-130 \pi 131 \pi 132 \pi$ & $115 \pi 117 \pi 120 \pi-130 \pi 131 \pi 132 \pi$ \\
\hline $7(116)$ & $108 \sigma 111 \sigma 115 \sigma-122 \sigma 128 \sigma 133 \sigma$ & $101 \sigma 109 \pi 111 \pi-122 \pi 124 \pi 126 \sigma$ & $110 \pi 111 \pi 115 \pi-122 \pi 171 \pi 173 \pi$ \\
\hline $9(104)$ & $97 \sigma 98 \sigma 102 \sigma-109 \sigma 110 \sigma 114 \sigma$ & $96 \pi 97 \pi 98 \pi-109 \pi 110 \pi 111 \pi$ & $97 \pi 98 \pi 102 \pi-109 \pi 110 \pi 116 \pi$ \\
\hline $10(104)$ & $97 \sigma 98 \sigma 102 \sigma-110 \sigma 112 \sigma 114 \sigma$ & $94 \pi 97 \pi 98 \pi-109 \pi 110 \pi 112 \sigma$ & $97 \pi 98 \pi 102 \pi-109 \pi 110 \pi 142 \pi$ \\
\hline $11(100)$ & $89 \sigma 94 \sigma 98 \sigma-106 \sigma 110 \sigma 111 \sigma$ & $89 \sigma 91 \pi 94 \pi-106 \pi 108 \pi 110 \sigma$ & $91 \pi 94 \pi 98 \pi-106 \pi 140 \pi 142 \pi$ \\
\hline $12(112)$ & $104 \sigma 106 \sigma 110 \sigma-118 \sigma 123 \sigma 124 \sigma$ & $99 \pi 104 \pi 106 \pi-118 \pi 120 \pi 121 \sigma$ & $106 \pi 107 \pi 110 \pi-118 \pi 124 \pi 164 \pi$ \\
\hline $13(120)$ & $112 \sigma 116 \sigma 120 \sigma-126 \sigma 127 \sigma 128 \sigma$ & $107 \pi 110 \pi 112 \pi-126 \pi 127 \pi 128 \pi$ & $110 \pi 112 \pi 116 \pi-126 \pi 127 \pi 128 \pi$ \\
\hline $14(112)$ & $105106111 \sigma-118 \sigma 123 \sigma 124 \sigma$ & $104 \pi 105 \pi 106 \pi-118 \pi 120 \pi 122 \sigma$ & $106 \pi 107 \pi 111 \pi-118 \pi 124 \pi 164 \pi$ \\
\hline $15(164)$ & $156 \sigma 159 \sigma 163 \sigma-169 \sigma 170 \sigma 171 \sigma$ & $141 \sigma 153 \pi 156 \pi-169 \pi 170 \pi 171 \pi$ & $153 \pi 156 \pi 159 \pi-169 \pi 170 \pi 171 \pi$ \\
\hline $17(156)$ & $145 \sigma 150 \sigma 151 \sigma-161 \sigma 172180$ & $132 \pi 147 \pi 150 \pi-161 \pi 163 \pi 165$ & $150 \pi 151 \pi 153 \pi-161 \pi 242 \pi 247 \pi$ \\
\hline $18(140)$ & $119 \sigma 134 \sigma 137 \sigma-145 \sigma 149 \sigma 159$ & $119 \pi 131 \pi 134 \pi-145 \pi 147 \pi 154 \sigma$ & $131 \pi 134 \pi 137 \pi-145 \pi 192 \pi 199 \pi$ \\
\hline 19 (112) & $99 \sigma 106 \sigma 110 \sigma-117 \sigma 127 \sigma 128 \sigma$ & $99 \pi 104 \pi 106 \pi-117 \pi 119 \pi 121 \sigma$ & $104 \pi 106 \pi 110 \pi-117 \pi 127 \pi 154 \sigma$ \\
\hline $20(136)$ & $128 \sigma 132 \sigma 135 \sigma-141 \sigma 142 \sigma 143 \sigma$ & $121 \sigma 126128 \pi-141 \pi 142 \pi 143 \pi$ & $130 \operatorname{lp} \pi 131 \pi 132 \pi-141 \pi 142 \pi 143 \pi$ \\
\hline $21(160)$ & $154 \sigma 155 \sigma 159 \sigma-165 \sigma 166 \sigma 167 \sigma$ & $143 \pi 148 \pi 152 \pi-165 \pi 166 \pi 167 \pi$ & $152154 \pi 155 \pi-165 \pi 166 \pi 167 \pi$ \\
\hline $22(140)$ & $120 \sigma 134 \sigma 137 \sigma-145 \sigma 148 \sigma 150 \sigma$ & $128 \pi 129 \pi 134 \pi-145 \pi 147 \pi 148 \pi$ & $129 \pi 134 \pi 137 \pi-145 \pi 211 \pi 213 \pi$ \\
\hline $23(152)$ & $142 \sigma 146 \sigma 149 \sigma-157 \sigma 162 \sigma 166 \sigma$ & $135 \pi 142 \pi 146 \pi-157 \pi 159 \pi 160 \pi$ & $142 \pi 146 \pi 149 \pi-157 \pi 166 \pi 234 \pi$ \\
\hline $24(136)$ & $115 \sigma 130 \sigma 133 \sigma-141 \sigma 146 \sigma 162 \sigma$ & $106 \sigma 123 \pi 130 \pi-141 \pi 143 \pi 144 \pi$ & $125 \pi 130 \pi 133 \pi-141 \pi 183 \sigma 189 \sigma$ \\
\hline $25(108)$ & $95 \sigma 102 \sigma 105 \sigma-113 \sigma 117 \sigma 118 \sigma$ & $95 \sigma 99 \pi 102 \pi-113 \pi 115 \pi 117 \sigma$ & $99 \pi 102 \pi 105 \pi-113 \pi 157 \pi 159 \pi$ \\
\hline $26(120)$ & $111 \sigma 114 \sigma 117 \sigma-125 \sigma 129 \sigma 130 \sigma$ & $110 \pi 111 \pi 114 \pi-125 \pi 127 \sigma 129 \sigma$ & $114 \pi 115 \pi 117 \pi-125 \pi 177 \pi 180 \sigma$ \\
\hline $27(128)$ & $120 \sigma 123 \sigma 127 \sigma-133 \sigma 134 \sigma 135 \sigma$ & $115 \pi 117 \pi 120 \pi-133 \pi 134 \pi 135 \pi$ & $120 \pi 122 \pi 123 \pi-133 \pi 134 \pi 135 \pi$ \\
\hline
\end{tabular}

\section{Discussion}

The HeLa inhibition mechanism is unknown. We have stated that "it is important to stress that our hypothesis covers multi-step (for example, in the $n$-th step molecules must cross a pore) and multimechanistic (for example, to cross the pore molecules must interact consecutively with $\mathrm{j}$ unknown sites) processes. Therefore it seems logical to state that a necessary condition to obtain good structure-activity relationships is that all the steps and all the mechanisms inside each step must be the same for all the group of molecules under study" [44]. If the molecules studied here employ multi-step and/or multimechanistic action mechanisms that are not exactly the same for all, we may expect that the linear multiple regression results contain sometimes variables whose interpretation seems contradictory.

The beta values shows that the importance of variables is $S_{21}^{E}>S_{16}^{N}>F_{21}(H O M O) *>Q_{16}>F_{20}(H O M O) *>$ $F_{15}(L U M O)^{*}>S_{22}^{N}(L U M O) *>F_{23}(H O M O) *$. The process seems to be charge and orbital-controlled. A variable-byvariable analysis indicates that a good activity is associated with low negative numerical values of $S_{21}^{E}$ (they are always negative) and $Q_{16}$, with low numerical values of $F_{21}(H O M O) *$ and $F_{20}(H O M O) *$ (their values are always positive) and with high numerical values for $F_{15}(L U M O)$ * and $F_{23}(H O M O)^{*}$. If $S_{22}^{N}(L U M O)^{*}$ is positive, a high inhibitory activity is associated with low numerical values. If $S_{16}^{N}$ is positive, a good activity is associated with low numerical values for this index.

Atom 21 is a carbon atom in the lateral chain of ring $\mathrm{C}$ (Figure 2). Table 6 shows that all local MO have $\sigma$ nature. A low value of $S_{21}^{E}$ indicates that atom 21 should interact with a sigma electron rich center through its empty sigma local MOs. Note that the local HOMO* and the local LUMO* do not coincide with the molecule's frontier MOs. The interactions can be of the $\sigma-\pi$ or $\sigma-\sigma$ kind. The $\sigma-\sigma$ interaction may occur with the sigma MOs of the $-\mathrm{CH}_{2}$ - groups of some amino acids. This coincides with the requirement of a low value for $F_{21}(H O M O)^{*}$. Atom 16 is a carbon atom in ring $\mathrm{C}$ (Figure 2). If $S_{16}^{N}$ is positive, a high inhibitory activity is associated with low numerical values for this index. From the 
definition of $S_{16}^{N}$, the dominant term is $S_{16}^{N}(L U M O)^{*}$. Low numerical values are obtained by shifting upwards the energy of the empty MOs, making this atom a bad electron acceptor. Therefore, we suggest that atom 16 is interacting with an electron deficient center. On the other hand, Eq. 16 shows that a high inhibitory activity is related with a positive value for $\mathrm{Q}_{16}$, fact that seems to be contradictory with the interaction with an electron deficient center. Examining Table II we may see that $S_{16}^{N}$ is more significant than $\mathrm{Q}_{16}$. Therefore, and as a first approximation, we shall not consider $\mathrm{Q}_{16}$. Atom 20 is a carbon atom of the side chain of ring $\mathrm{C}$ (Figure 2). All local MOs have $\sigma$ nature (Table 6). A low value for $F_{20}(H O M O) *$ suggests that atom 20 is probably interacting with a center rich in sigma electrons. Note that this condition is the same that the one for atom 21 . Atom 22 is the carbon atom of the carboxylate moiety of the side chain of ring $\mathrm{C}$ (Figure 2). (LUMO) ${ }_{22}{ }^{*}$ is a $\pi \mathrm{MO}$ in all molecules (Table 6). A low value for $S_{22}^{N}(L U M O) *$ is associated with high inhibitory activity. This value is obtained by shifting upwards the $(L U M O)_{22}$ * eigenvalue and making the MO less reactive. So, we suggest that atom 22 is interacting with a $\pi$ electron deficient center. Atom 15 is a carbon atom in ring $\mathrm{C}$ (Figure 2). $(L U M O)_{15} *$ is a $\mathrm{MO}$ of $\pi$ nature (Table 5). A high value for $F_{15}(L U M O) *$ suggests that this lowest unoccupied local MO is interacting with an electron rich center. Atom 23 is an oxygen atom of the carboxylate moiety in the side chain of ring $\mathrm{C}$ (Figure 2). A high value for $F_{23}(H O M O)^{*}$ suggests that the highest occupied local is interacting with an electron deficient center. All the above suggestions are shown in the partial 2D pharmacophore of Figure 4.

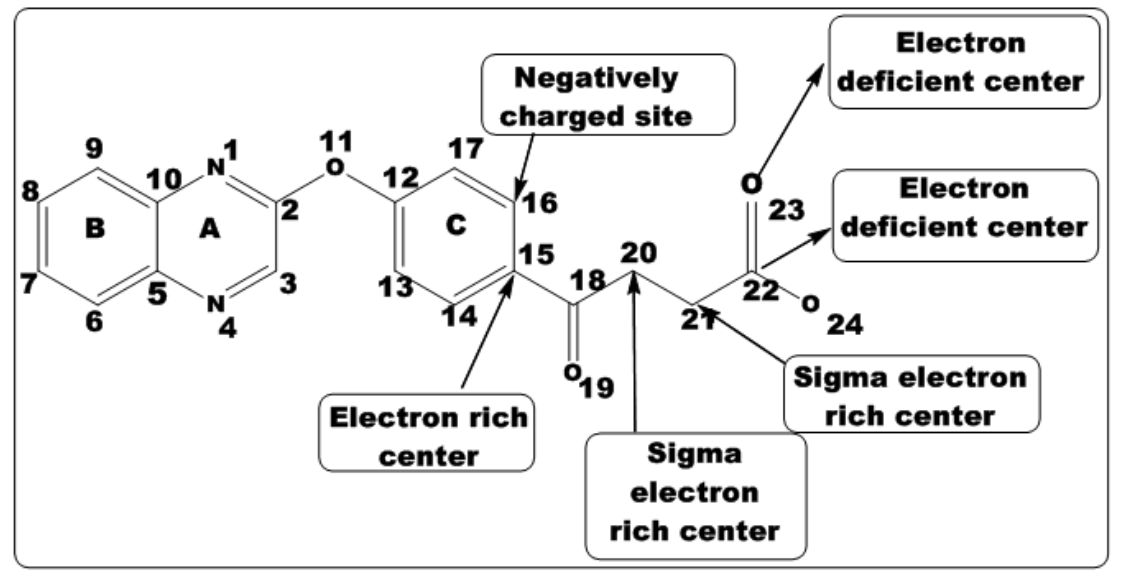

Figure 4. Partial 2D pharmacophore for the anti-proliferative activities of quinoxaline derivatives on the HeLa cell line.

\section{Conclusion}

We obtained a statistically significant relationship between the variation of the anti-proliferative activity of some quinoxaline derivatives and the variation of the numerical values of a set of local atomic reactivity indices. This allowed us to build the associated pharmacophore that should serve as a starting point for chemical modifications producing more active compounds. According to the obtained pharmacophore, it is not necessary to modify the indices of the atoms of the quinoxaline cycle. But the indices which would be modified to improve the anti-proliferative activity are those from the side chain.

\section{References}

[1] Skloot, R., The Immortal Life of Henrietta Lacks, Pan Macmillan, 2011.

[2] Scherer, W. F., Syverton, J. T., Gey, G. O., Studies on the propagation in vitro of poliomyelitis viruses. IV. Viral multiplication in a stable strain of human malignant epithelial cells (strain HeLa) derived from an epidermoid carcinoma of the cervix. J. Exp. Med. 1953, 97, 695-710.
[3] Landry, J. J. M., Pyl, P. T., Rausch, T., Zichner, T., et al., The Genomic and Transcriptomic Landscape of a HeLa Cell Line. G3 Genes Genomes Genet. 2013, 3, 1213-1224.

[4] Akhtar, J., Khan, A. A., Ali, Z., Haider, R., et al., Structureactivity relationship (SAR) study and design strategies of nitrogen-containing heterocyclic moieties for their anticancer activities. Eur. J. Med. Chem. 2017, 125, 143-189.

[5] Akrami, H., Safavi, M., Mirjalili, B. F., Ashkezari, M. D., et al., Facile synthesis and antiproliferative activity of $7 \mathrm{H}-$ benzo[7,8]chromeno[2,3-d]pyrimidin-8-amines. Eur. J. Med. Chem. 2017, 127, 128-136.

[6] An, W., Wang, W., Yu, T., Zhang, Y., et al., Discovery of novel 2-phenyl-imidazo[1,2-a]pyridine analogues targeting tubulin polymerization as antiproliferative agents. Eur. J. Med. Chem. 2016, 112, 367-372.

[7] Hernández-Padilla, L., Vázquez-Rivera, D., Sánchez-Briones, L. A., Díaz-Pérez, A. L., et al., The Antiproliferative Effect of Cyclodipeptides from Pseudomonas aeruginosa PAO1 on HeLa Cells Involves Inhibition of Phosphorylation of Akt and S6k Kinases. Molecules 2017, 22.

[8] Carta, D., Bortolozzi, R., Sturlese, M., Salmaso, V., et al., Synthesis, structure-activity relationships and biological evaluation of 7-phenyl-pyrroloquinolinone 3-amide derivatives as potent antimitotic agents. Eur. J. Med. Chem. 2017, 127, 643-660. 
[9] Cheng, W.-H., Shang, H., Niu, C., Zhang, Z.-H., et al., Synthesis and Evaluation of New Podophyllotoxin Derivatives with in Vitro Anticancer Activity. Molecules 2015, 20, 1226612279 .

[10] Dandriyal, J., Singla, R., Kumar, M., Jaitak, V., Recent developments of C-4 substituted coumarin derivatives as anticancer agents. Eur. J. Med. Chem. 2016, 119, 141-168.

[11] Diao, P.-C., Li, Q., Hu, M.-J., Ma, Y.-F., et al., Synthesis and biological evaluation of novel indole-pyrimidine hybrids bearing morpholine and thiomorpholine moieties. Eur. J. Med. Chem. 2017, 134, 110-118.

[12] Li, F.-Y., Wang, X., Duan, W.-G., Lin, G.-S., Synthesis and In Vitro Anticancer Activity of Novel Dehydroabietic AcidBased Acylhydrazones. Molecules 2017, 22.

[13] Fytas, C., Zoidis, G., Tsotinis, A., Fytas, G., et al., Novel 1-(2aryl-2-adamantyl)piperazine derivatives with antiproliferative activity. Eur. J. Med. Chem. 2015, 93, 281-290.

[14] Gabr, M. T., El-Gohary, N. S., El-Bendary, E. R., El-Kerdawy, M. M., et al., Isatin- $\beta$-thiocarbohydrazones: Microwaveassisted synthesis, antitumor activity and structure-activity relationship. Eur. J. Med. Chem. 2017, 128, 36-44.

[15] Gonçalves, B. M. F., Salvador, J. A. R., Marín, S., Cascante, M., Synthesis and anticancer activity of novel fluorinated asiatic acid derivatives. Eur. J. Med. Chem. 2016, 114, 101117 .

[16] Li, W., Tan, G., Cheng, J., Zhao, L., et al., A Novel Photosensitizer 31,131-phenylhydrazine -Mppa (BPHM) and Its in Vitro Photodynamic Therapy against HeLa Cells. Molecules 2016, 21.

[17] Żołnowska, B., Sławiński, J., Pogorzelska, A., Szafrański, K., et al., Novel 5-Substituted 2-(Aylmethylthio)-4-chloro-N-(5aryl-1,2,4-triazin-3-yl)benzenesulfonamides: Synthesis, Molecular Structure, Anticancer Activity, Apoptosis-Inducing Activity and Metabolic Stability. Molecules 2016, 21.

[18] Liu, Q., Li, W., Sheng, L., Zou, C., et al., Design, synthesis and biological evaluation of novel asperphenamate derivatives. Eur. J. Med. Chem. 2016, 110, 76-86.

[19] Romagnoli, R., Baraldi, P. G., Prencipe, F., Oliva, P., et al., Design, synthesis and biological evaluation of 3-substituted-2oxindole hybrid derivatives as novel anticancer agents. Eur. $J$. Med. Chem. 2017, 134, 258-270.

[20] Levrier, C., Sadowski, M. C., Rockstroh, A., Gabrielli, B., et al., $6 \alpha$-Acetoxyanopterine: A Novel Structure Class of Mitotic Inhibitor Disrupting Microtubule Dynamics in Prostate Cancer Cells. Mol. Cancer Ther. 2017, 16, 3-15.

[21] Sun, B., Li, L., Hu, Q., Zheng, H., et al., Design, synthesis, biological evaluation and molecular modeling study of novel macrocyclic bisbibenzyl analogues as antitubulin agents. Eur. J. Med. Chem. 2017, 129, 186-208.

[22] Xie, R., Yao, Y., Tang, P., Chen, G., et al., Design, synthesis and biological evaluation of novel hydroxamates and 2aminobenzamides as potent histone deacetylase inhibitors and antitumor agents. Eur. J. Med. Chem. 2017, 134, 1-12.

[23] Xia, Q.-H., Hu, W., Li, C., Wu, J.-F., et al., Design, synthesis, biological evaluation and molecular docking study on peptidomimetic analogues of XK469. Eur. J. Med. Chem. 2016, 124, 311-325.
[24] Banu, S., Bollu, R., Bantu, R., Nagarapu, L., et al., Design, synthesis and docking studies of novel 1,2-dihydro-4hydroxy-2-oxoquinoline-3-carboxamide derivatives as a potential anti-proliferative agents. Eur. J. Med. Chem. 2017, $125,400-410$.

[25] Gregorić, T., Sedić, M., Grbčić, P., Paravić, A. T., et al., Novel pyrimidine-2,4-dione-1,2,3-triazole and furo[2,3d]pyrimidine-2-one-1,2,3-triazole hybrids as potential anticancer agents: Synthesis, computational and X-ray analysis and biological evaluation. Eur. J. Med. Chem. 2017, 125, $1247-1267$

[26] Karki, R., Jun, K.-Y., Kadayat, T. M., Shin, S., et al., A new series of 2-phenol-4-aryl-6-chlorophenyl pyridine derivatives as dual topoisomerase I/II inhibitors: Synthesis, biological evaluation and 3D-QSAR study. Eur. J. Med. Chem. 2016, $113,228-245$.

[27] Pogorzelska, A., Sławiński, J., Żołnowska, B., Szafrański, K., et al., Novel 2-(2-alkylthiobenzenesulfonyl)-3-(phenylprop-2ynylideneamino)guanidine derivatives as potent anticancer agents - Synthesis, molecular structure, QSAR studies and metabolic stability. Eur. J. Med. Chem. 2017, 138, 357-370.

[28] Grozav, A., Porumb, I.-D., Găină, L. I., Filip, L., et al., Cytotoxicity and Antioxidant Potential of Novel 2- $(2-((1 \mathrm{H}-$ indol-5yl)methylene)-hydrazinyl)-thiazole Derivatives. Molecules 2017, 22.

[29] Gómez-Jeria, J. S., Abarca-Martínez, S., A theoretical approach to the cytotoxicity of a series of $\beta$ carbolinedithiocarbamate derivatives against prostatic cancer (DU-145), breast cancer (MCF-7), human lung adenocarcinoma (A549) and cervical cancer (HeLa) cell lines. Pharma Chem. 2016, 8, 507-526.

[30] Gómez-Jeria, J. S., 45 Years of the KPG Method: A Tribute to Federico Peradejordi. J. Comput. Methods Mol. Des. 2017, 7, 17-37.

[31] Hudson, R. F., Klopman, G., A general perturbation treatment of chemical reactivity. Tetrahedron Lett. 1967, 8, 1103-1108.

[32] Klopman, G., Chemical reactivity and the concept of chargeand frontier-controlled reactions. J. Am. Chem. Soc. 1968, 90, 223-234.

[33] Klopman, G., Hudson, R. F., Polyelectronic perturbation treatment of chemical reactivity. Theor. Chim. Acta 1967, 8, 165-174.

[34] Peradejordi, F., Martin, A. N., Cammarata, A., Quantum chemical approach to structure-activity relationships of tetracycline antibiotics. J. Pharm. Sci. 1971, 60, 576-582.

[35] Gómez Jeria, J. S., A new set of local reactivity indices within the Hartree-Fock-Roothaan and density functional theory frameworks. Can. Chem. Trans. 2013, 1, 25-55.

[36] Gómez Jeria, J. S., Elements of Molecular Electronic Pharmacology, 1st ed., Ediciones Sokar, Santiago de Chile 2013.

[37] Gómez Jeria, J. S., Calculation of the Nucleophilic Superdelocalizability by the CNDO/2 Method. J. Pharm. Sci. 1982, 71, 1423-1424.

[38] Gómez Jeria, J. S., La Pharmacologie Quantique. Boll Chim Farm. 1982, 121, 619-625. 
[39] Gomez-Jeria, J. S., On some problems in quantum pharmacology I. The partition functions. Int. J. Quantum Chem. 1983, 23, 1969-1972.

[40] Gomez-Jeria, J. S., Cassels, B. K., Saavedra-Aguilar, J. C., A quantum-chemical and experimental study of the hallucinogen $( \pm)$-1-(2,5-dimethoxy-4-nitrophenyl)-2-aminopropane (DON). Eur. J. Med. Chem. 1987, 22, 433-437.

[41] Gomez-Jeria, J. S., Morales-Lagos, D., Rodriguez-Gatica, J. I., Saavedra-Aguilar, J. C., Quantum-chemical study of the relation between electronic structure and pA2 in a series of 5substituted tryptamines. Int. J. Quantum Chem. 1985, 28, 421-428.

[42] Gómez-Jeria, J. S., Modeling the Drug-Receptor Interaction in Quantum Pharmacology, in: Maruani, J. (Ed.), Molecules in Physics, Chemistry, and Biology, Springer Netherlands, Dordrecht 1989, pp. 215-231.

[43] Gomez-Jeria, J. S., Sotomayor, P., Quantum chemical study of electronic structure and receptor binding in opiates. J. Mol. Struct. THEOCHEM 1988, 166, 493-498.

[44] Gómez Jeria, J. S., Flores-Catalán, M., Quantum-chemical Modeling of the Relationships between Molecular Structure and In Vitro Multi-Step, Multimechanistic Drug Effects. HIV1 Replication Inhibition and Inhibition of Cell Proliferation as Examples. Can. Chem. Trans. 2013, 1, 215-237.

[45] Gómez Jeria, J. S., The use of competitive ligand binding results in QSAR studies. Il Farm. n. d., 40, 299-302.

[46] Bruna-Larenas, T., Gómez-Jeria, J. S., A DFT and Semiempirical Model-Based Study of Opioid Receptor Affinity and Selectivity in a Group of Molecules with a Morphine Structural Core. Int. J. Med. Chem. 2012, 16.

[47] Gómez-Jeria, J. S., Castro-Latorre, P., A Density Functional Theory analysis of the relationships between the Badger index measuring carcinogenicity and the electronic structure of a series of substituted Benz[a]anthracene derivatives, with a suggestion for a modified carcinogenicity index. Chem. Res. J. 2017, 2, 112-126.

[48] Gómez Jeria, J. S., Ovando-Guerrero, R., A DFT Study of the Relationships between Electronic Structure and Central Benzodiazepine Receptor Affinity in a group of Imidazo[1,5a]quinoline derivatives and a group of 3-Substituted 6-Phenyl4H-imidazo[1,5-a]-[1,4]benzodiazepines and related compounds. Chem. Res. J. 2017, 2, 170-181.

[49] Kpotin, A. G., Atohoun, G. S., Kuevi, A. U., Houngue-Kpota, A., et al., A quantum-chemical study of the relationships between electronic structure and anti- HIV-1 activity of a series of HEPT derivatives. J. Chem. Pharm. Res. 2016, 8, 1019-1026.

[50] Kpotin, G., Atohoun, S. Y. G., Kuevi, A. U., Kpota-Hounguè, A., et al., A Quantum-Chemical study of the Relationships between Electronic Structure and Trypanocidal Activity against Trypanosoma Brucei Brucei of a series of Thiosemicarbazone derivatives. Pharm. Lett. 2016, 8, 215222.

[51] Gómez-Jeria, J. S., Moreno-Rojas, C., Dissecting the drugreceptor interaction with the Klopman-Peradejordi-Gómez (KPG) method. I. The interaction of 2,5dimethoxyphenethylamines and their N-2-methoxybenzylsubstituted analogs with 5-HT1A serotonin receptors. Chem. Res. J. 2017, 2, 27-41.

[52] Kpotin, A. G., Kankinou, G., Kuevi, U., Gómez Jeria, J. S., et al., A Theoretical Study of the Relationships between Electronic Structure and Inhibitory Effects of Caffeine Derivatives on Neoplastic Transformation. Int. Res. J. Pure Appl. Chem. 2017, 14, 1-10.

[53] Robles-Navarro, A., Gómez Jeria, J., A quantum-chemical analysis of the relationships between electronic structure and cytotoxicity, GyrB inhibition, DNA supercoiling inhibition and antitubercular activity of a series of quinolineaminopiperidine hybrid analogues. Pharma Chem. 2016, 8, 417-440.

[54] Frisch, M. J., Trucks, G. W., Schlegel, H. B., Scuseria, G. E., et al., G03 Rev. E. 01, Gaussian:, Pittsburgh, PA, USA 2007.

[55] Gómez Jeria, J. S., D-Cent-QSAR: A program to generate Local Atomic Reactivity Indices from Gaussian 03 log files. 1.0, Santiago de Chile 2014.

[56] Gómez-Jeria, J. S., An empirical way to correct some drawbacks of Mulliken Population Analysis (Erratum in: J. Chil. Chem. Soc., 55, 4, IX, 2010). J. Chil. Chem. Soc. 2009, $54,482-485$.

[57] Gómez Jeria, J. S., Tables of proposed values for the Orientational Parameter of the Substituent. II. Res. J. Pharm. Biol. Chem. Sci. 2016, 7, 2258-2260.

[58] Gómez Jeria, J. S., Tables of proposed values for the Orientational Parameter of the Substituent. I. Monoatomic, Diatomic, Triatomic, n-CnH2n+1, O-n-CnH2n+1, NRR', and Cycloalkanes (with a single ring) substituents. Res. J. Pharm. Biol. Chem. Sci. 2016, 7, 288-294.

[59] Statsoft, Statistica 8.0, 2300 East 14 th St. Tulsa, OK 74104, USA 1984. 